This is an electronic reprint of the original article. This reprint may differ from the original in pagination and typographic detail.

Author(s): Banichuk, Nikolay; Kurki, Matti; Neittaanmäki, Pekka; Saksa, Tytti; Tirronen, Maria; Tuovinen, Tero

Title: $\quad$ Optimization and analysis of processes with moving materials subjected to fatigue fracture and instability

Year: $\quad 2013$

Version:

Please cite the original version:

Banichuk, N., Kurki, M., Neittaanmäki, P., Saksa, T., Tirronen, M., \& Tuovinen, T. (2013). Optimization and analysis of processes with moving materials subjected to fatigue fracture and instability. Mechanics Based Design of Structures and Machines: An International Journal, 41(2), 146-167. https://doi.org/10.1080/15397734.2012.708630

All material supplied via JYX is protected by copyright and other intellectual property rights, and duplication or sale of all or part of any of the repository collections is not permitted, except that material may be duplicated by you for your research use or educational purposes in electronic or print form. You must obtain permission for any other use. Electronic or print copies may not be offered, whether for sale or otherwise to anyone who is not an authorised user. 


\title{
Optimization and Analysis of Processes with Moving Materials Subjected to Fatigue Fracture and Instability
}

\author{
Nikolay Banichuk ${ }^{a, b} \quad$ Matti Kurki $^{a, c} \quad$ Pekka Neittaanmäki $^{a}$ \\ Tytti Saksa $^{a, *} \quad$ Maria Tirronen ${ }^{a} \quad$ Tero Tuovinen $^{a}$
}

${ }^{a}$ Department of Mathematical Information Technology, P.O. Box 35 (Agora),

FI-40014 University of Jyväskylä, Finland

${ }^{b}$ Ishlinsky Institute for Problems in Mechanics RAS, Prospect Vernadskogo 101, bld. 1, 119526 Moscow, Russian Federation

${ }^{c}$ School of Technology, JAMK University of Applied Sciences, P.O. Box 207,

FI-40101 Jyväskylä, Finland

E-mail addresses: banichuk@ipmnet.ru, matti.kurki@jamk.fi, pekka.neittaanmaki@jyu.fi, tytti.saksa@jyu.fi, maria.tirronen@gmail.com, tero.tuovinen@jyu.fi

${ }^{*}$ Corresponding author. Tel.: +358 40805 3296. Fax: +358 142602771 


\begin{abstract}
We study safe conditions and process effectiveness of systems of moving materials from the viewpoint of failures including fracture and loss of stability. The web is modelled as a thin elastic plate made of brittle material, travelling between a system of supports at a constant velocity, and subjected to bending, in-plane tension and small initial cracks. We study crack growth under cyclic in-plane tension and transverse buckling of the web analytically. We seek optimal in-plane tension that maximizes a performance vector function consisting of the number of cycles before fracture, the critical velocity and process effectiveness. The present way of applying optimization in the studies of fracture and stability is new and affords an analytical tool for process analysis techniques.
\end{abstract}

Keywords Moving materials; Fatigue fracture; Stability; Multi-objective optimization; Productivity

\title{
1 Introduction
}

In many applications of axially moving materials, such as paper making processes, printing presses, manufacturing of plastic films and sheets, and extrusion of aluminium foil, textiles and other materials, there is a demand for driving or running the system fast and, at the same time, avoiding damages. It is known that, in such systems, an increase in tension has a stabilizing effect but a decrease in tension may lead to a loss of stability. From the viewpoint of fracture, tension has an opposite effect: high tension may lead to growing or arising of cracks, and tension low enough then guarantees safe conditions.

By avoiding failures, we try to achieve high productivity or effectiveness of such long processes in which the produced material travels between supports. The productivity depends 
mainly on the material velocity and the structural longevity. By longevity or structural longevity, we refer to the number of load cycles that the (cracked) material sustains before fracture failure. Introducing a new idea of combining the stability and fatigue fracture analyses, we start with such theoretical models that provide explicit analytical solutions. More complicated theoretical models can be used instead, resulting maybe in more accurate results but definitely in slower solution processes. We concentrate especially on paper making productivity in which avoiding web breaks is crucial. Web breaks have concerned many scientists, e.g., Valenzuela et al. (2009) who developed a computer-based setting for drive controllers in paper machines to help to avoid web breaks. However, the analysis that we will proceed is also applicable to any other analogous processes.

The stability of axially moving materials has been studied modelling the web as a string, beam, membrane or plate, and the critical conditions have been examined with the help of dynamic analysis of frequency spectrum. Stability of travelling rectangular membranes and plates was first studied by Ulsoy and Mote (1982), and Lin and Mote $(1995,1996)$.

Archibald and Emslie (1958), and Simpson (1973) studied the effects of axial motion on the frequency spectrum and eigenfunctions. It was shown that the travelling string and beam both experience divergence instability at a sufficiently high speed, which is called the critical speed. Dynamics and stability considerations of moving materials were reviewed by Mote (1972) and Wickert and Mote (1988), who also have studied stability of axially moving strings and beams (Wickert and Mote, 1990). Wang et al. (2005) recently showed that for the transverse motion of a string, no static instability occurs at a critical velocity.

Stability of out-of-plane vibrations of axially moving rectangular membranes was studied by Shin et al. (2005). For the behaviour of the membrane, it was found that the motion is stable until a critical speed, at which statical instability occurs. In the recent studies 
concerning axially moving plates, material properties such as orthotropicity (Banichuk et al., 2011a) or viscoelasticity (Marynowski, 2010) have been taken into consideration and their effects on the plate behaviour have been investigated. Also such phenomena as winding in the context of axially moving materials has been studied (Garziera and Amabili, 2000).

Lin (1997) studied stability of axially moving plates, and numerically showed that loss of stability of the plate occurs in a form of divergence at a sufficiently high speed. The critical velocity and the corresponding critical shapes of an axially moving elastic plate were studied, and an analytical expression for the critical velocity was provided by Banichuk et al. (2010a).

The field of fracture mechanics was developed by Irwin (1958), based on the early papers of Inglis (1913), Griffith (1921) and Westergaard (1939). Linear elastic fracture mechanics was first applied to paper materials by Seth and Page (1974), who measured fracture toughness for different paper materials. Swinehart and Broek (1995) determined the fracture toughness of paper using both the stress intensity factor and the strain energy release rate. They found that the measured crack length and fracture toughness were in a good agreement with the LEFM theory.

Wathén (2003) discussed how the damages in paper affect the web breaks in paper making. Tryding (1996) has studied crack growth evolution in paper material using experiments and a cohesive crack model with finite element analysis. Fatigue of wood-pulp fibres on micro-structural level has been studied by Hamad (1997, 1998).

Previously in the context of web vibrations, fracture has been included into the problem dynamics and the effects of the cracks on the stability has been studied. Various analyses of vibrations and stability of stationary beams and plates exists in the literature. An extensive review on fracture of cracked materials and challenges in such models was discussed by 
Dimarogonas (1996). Finite element analysis has often been applied to analyse the vibrations and stability of cracked rectangular plates, considering centre or edge located cracks. Bachene et al. (2009) and used the extended finite element method and Liew et al. (1994) developed an efficient decomposition method to study vibrations of cracked plates. Brighenti (2005) examined buckling failure of cracked plates for different crack orientations with the help of finite element analysis. Both buckling and vibration analysis were covered in the finite element studies of cracked plates by Prabhakara and Datta $(1993,1997)$.

Stahl and Keer (1972) studied vibrations and stability of rectangular plates with the help of dual series equations. Vafai et al. (2002) studied parametric instability of plates having one crack at an edge. They considered simply supported rectangular plates under periodic loadings using an integral equation method. Effects of cracks on the eigenfrequencies and eigenmodes of axially moving beams at sub-critical transport speeds was studied by Murphy and Zhang (2000). However, the effect of the cracks on the results was found to be small.

We assume the material to undergo cyclic loading (and unloading) in the processes and to contain small initial cracks at the free band (web) edges. The procedure of crack growth under cyclic loading is a widely studied area known as fatigue crack growth. Paris and Erdogan (1963) presented a law for the crack growth rate, which is called Paris' law. Paris' law assumes linear elastic fracture mechanics which in turn assumes a small plastic zone ahead of the crack tip (Irwin, 1958).

As mentioned above, optimal magnitude of tension is essential for safe conditions in systems with axially travelling material. Seeking the optimal tension but having several objectives, such as high structural longevity, transport velocity and productivity, we encounter a multi-objective optimization problem, which usually has no unique optimal solution but a set of "equally optimal", Pareto optimal results. Extensive literature reviews on multi-objective 
optimization are provided by White (1990) and Miettinen (1999). For a historical review of the origin and development of multi-criteria optimization, we refer to Stadler (1979). Surveys of concepts and methods of multi-objective optimization have also been done, e.g., by Chankong and Haimes (1983) and Steuer (1986). Optimization under constraints on material fracture has been done, e.g., by Banichuk et al. (2003), in which probabilistic-guaranteed approach was applied to optimal design of membrane shells under quasi-brittle fracture.

In this study, we apply optimization to analyses of stability and fracture. To our knowledge, theories of fracture and stability have not been combined before by using optimization. We study crack growth under cyclic in-plane tension and transverse buckling of the web analytically, which allows us extremely efficient solution methods for the use of real time computing. We derive a multi-objective optimization problem consisting of maximizing the critical web (band) velocity, the longevity (critical number of loading cycles) and the productivity with respect to the value of in-plane tension. The obtained objective vector function is transformed into a scalar objective function using the weighting method. For several important sub-problems, the optimal value of tension is found analytically in the Pareto sense with respect to the other problem parameters. The general results with dimensionless formulation are represented. Some examples using parameter values typical of paper materials are shown.

\section{Problem Set-Up}

Consider a web (continuum) travelling at a constant velocity between a long system of supports (rollers). The web undergoes open draws between the supports, between which the distances are assumed to be equal. The web is assumed to be tensioned and subjected 
to small tension variations during the process. Tension variations may arise due to Earth's gravity (Banichuk et al., 2011b), machine fluctuations or paper variability (Hristopulos and Uesaka, 2002).

We model the web as a plate with infinite length travelling at a constant speed $V_{0}$ in the $x$ direction and the supporting rollers to be located at $x=0, \ell, 2 \ell, 3 \ell, \ldots$ See Fig. 1 .
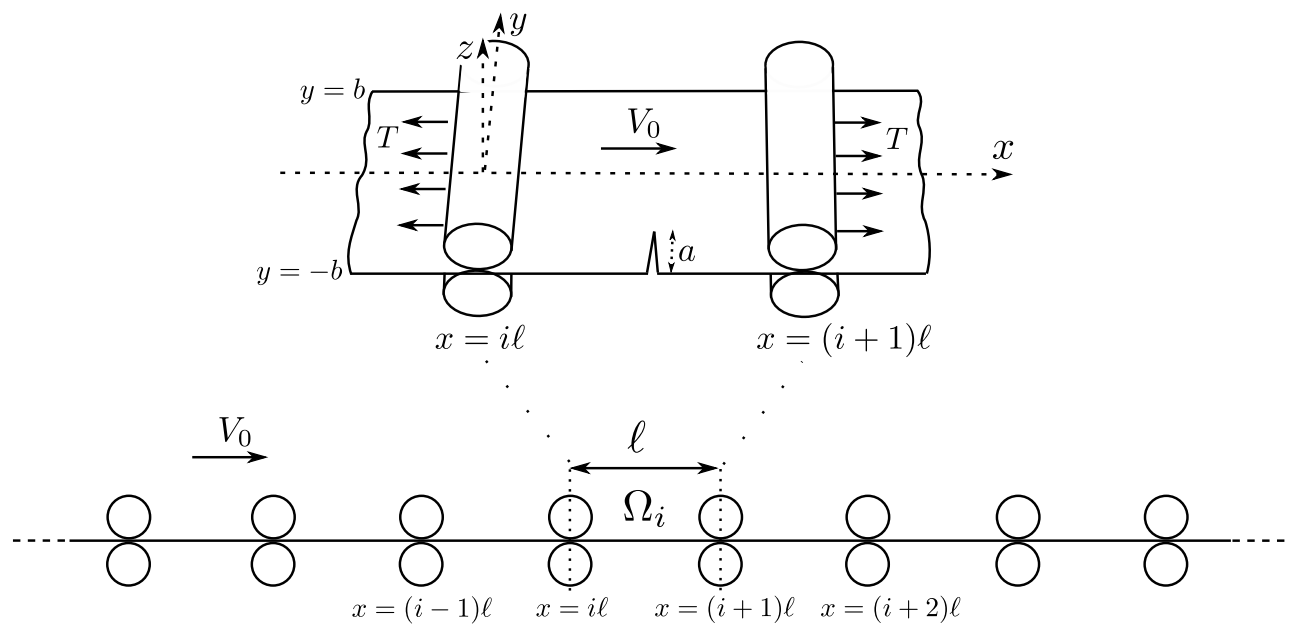

Figure 1: A travelling web having an initial crack, and being supported by a system of rollers.

We denote a rectangular part of the plate as follows:

$$
\Omega_{i}=\{(x, y): \quad i \ell \leq x \leq(i+1) \ell, \quad-b \leq y \leq b\}, \quad i=1,2,3, \ldots
$$

where $\ell$ and $b$ are prescribed geometric parameters. The considered plate is assumed to have constant thickness $h$, Poisson ratio $\nu$, Young modulus $E$, bending rigidity $D$, and mass per unit area (of the middle surface of the plate) $m$.

All the plate elements $\Omega_{i}$ are subjected to homogeneous (in the $y$ direction) tension $T$ 
acting in the $x$ direction. The sides

$$
\begin{aligned}
& \Gamma_{i, \ell}=\{x=i \ell, \quad-b \leq y \leq b\} \quad \text { and } \\
& \Gamma_{i, r}=\{x=(i+1) \ell, \quad-b \leq y \leq b\}
\end{aligned}
$$

are simply supported and the sides

$$
\begin{aligned}
& \Gamma_{i,-}=\{y=-b, \quad i \ell \leq x \leq(i+1) \ell\} \quad \text { and } \\
& \Gamma_{i,+}=\{y=b, \quad i \ell \leq x \leq(i+1) \ell\}
\end{aligned}
$$

are free of traction. Tension is supposed to be cyclic such that the web may undergo few or many cycles on each span. See Fig. 2.

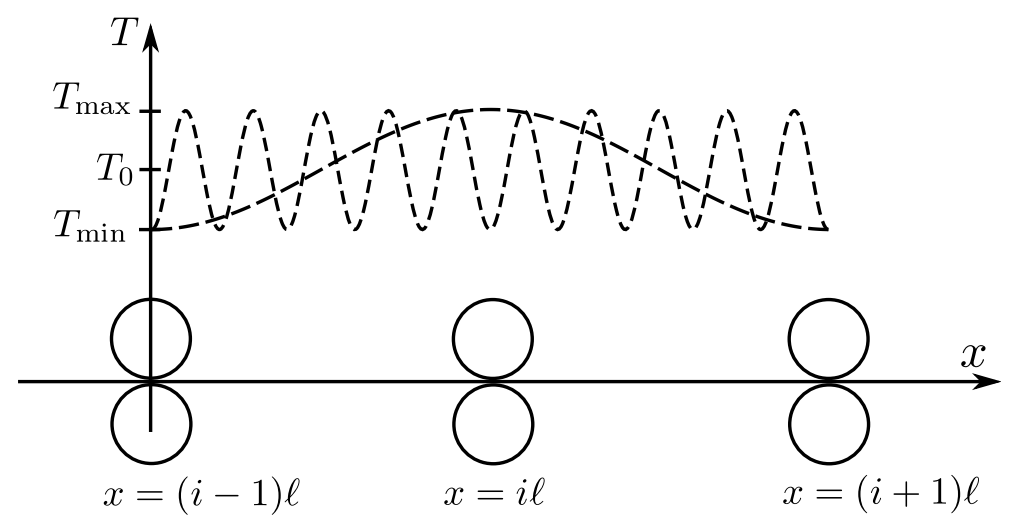

Figure 2: Two examples of cyclic tension. There may be few or many tension cycles per span. 


\section{Stability of Transverse Vibrations of the Web and Critical Velocity}

The critical velocity corresponding to the loss of stability of the transverse vibrations of the web is analysed here by the linearised Kirchhoff plate theory, in which we assume that

the transverse vibrations are small (Timoshenko and Woinowsky-Krieger, 1959). The critical velocity for each span $\Omega_{i}$ can be found by solving the buckling problem for dynamic transverse deflections of the plate (see Banichuk et al., 2010a, or Lin, 1997).

Stationary equations describing the behaviour of the web with the applied boundary conditions form the following eigenvalue problem (a buckling problem):

$$
\begin{array}{lr}
\left(m V_{0}^{2}-T_{0}\right) \frac{\partial^{2} w}{\partial x^{2}}+D\left(\frac{\partial^{4} w}{\partial x^{4}}+2 \frac{\partial^{4} w}{\partial x^{2} \partial y^{2}}+\frac{\partial^{4} w}{\partial y^{4}}\right)=0, & \text { in } \Omega_{i} \\
w=0, \frac{\partial^{2} w}{\partial x^{2}}=0, & \text { on } \Gamma_{i, \ell} \text { and } \Gamma_{i, r}, \\
\frac{\partial^{2} w}{\partial y^{2}}+\nu \frac{\partial^{2} w}{\partial x^{2}}=0, & \text { on } \Gamma_{i,-} \text { and } \Gamma_{i,+}, \\
\frac{\partial^{3} w}{\partial y^{3}}+(2-\nu) \frac{\partial^{3} w}{\partial x^{2} \partial y}=0, & \text { on } \Gamma_{i,-} \text { and } \Gamma_{i,+},
\end{array}
$$

where $D=E h^{3} /\left(12\left(1-\nu^{2}\right)\right)$, and we denote the eigenvalue

$$
\lambda=\gamma^{2}=\frac{\ell^{2}}{\pi^{2} D}\left(m V_{0}^{2}-T_{0}\right)
$$

The travelling plate subjected to a constant tension experiences divergence instability at a critical speed (Banichuk et al., 2010a)

$$
\left(V_{0}^{\mathrm{cr}}\right)^{2}=\frac{T_{0}}{m}+\frac{\gamma_{*}^{2}}{m} \frac{\pi^{2} D}{\ell^{2}}
$$




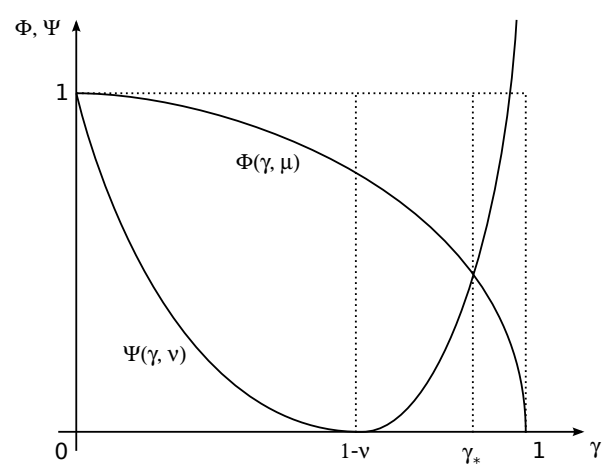

Figure 3: Behaviour of $\Phi$ and $\Psi$ as functions of $\gamma$.

where $\gamma_{*}^{2}=\lambda_{*}$ is the minimal eigenvalue of problem (1). Parameter $\gamma=\gamma_{*}$ is found as the root of the equation (see also Fig. 3)

$$
\Phi(\gamma, \mu)-\Psi(\gamma, \nu)=0
$$

where

$$
\begin{aligned}
& \Phi(\gamma, \mu)=\tanh \left(\frac{\sqrt{1-\gamma}}{\mu}\right) \operatorname{coth}\left(\frac{\sqrt{1+\gamma}}{\mu}\right), \\
& \Psi(\gamma, \nu)=\frac{\sqrt{1+\gamma}}{\sqrt{1-\gamma}} \frac{(\gamma+\nu-1)^{2}}{(\gamma-\nu+1)^{2}}, \quad \mu=\frac{\ell}{\pi b} .
\end{aligned}
$$

As it is seen from $(3)-(4)$, the root $\gamma=\gamma_{*}$ depends on $\nu$ and $\mu$ and does not depend on the other problem parameters, including the value of tension $T_{0}$. Consequently, the critical instability velocity, defined in (2), is increased with the increasing of tension $T_{0}$. However, increasing of $T_{0}$ is limited due to initial damages and other imperfections. 


\section{Fracture Under Constant Tension}

Assume that the value of tension is constant $T=T_{0}$ and that the plate has small surface cracks that arise at the free boundaries of the plate and have length $a$ with upper bound $a^{*}$, i.e.,

$$
a \leq a^{*} \ll 2 b,
$$

where $a^{*}$ is a given admissible value. The cracks are assumed to be orthogonal to the boundary lines, and thus, the external loading mode is an opening mode and cracks are considered in the $x y$ plane. If distances between the cracks are large enough, we may consider only one isolated crack of a limited length $a=a^{*}$. This is to say that there is no correlation between the singular stress fields that arise nearby crack ends.

The stress intensity factor $K$ can be expressed as (see Irwin, 1958, or Westergaard, 1939)

$$
K=\beta \sigma \sqrt{\pi a}=\beta \frac{T}{h} \sqrt{\pi a} .
$$

Here $\beta=1.12$ is a geometric factor $\left(\frac{a}{2 b}\right.$ is small) and $\sigma=\sigma_{x}$ is a component of a stress tensor. We express the brittle fracture condition as

$$
K=K_{\mathrm{C}},
$$

where $K_{\mathrm{C}}$ is the fracture toughness of material.

Consider now that the biggest admissible crack length $a^{*}$ coincides with the critical crack length $a^{\text {cr }}$, i.e.

$$
a^{*}=a^{\mathrm{cr}} .
$$


In this case $\left(T=T_{0}\right)$, by definition of $K_{\mathrm{C}}$, we have

$$
K_{\mathrm{C}}=K_{a=a^{\mathrm{cr}}}=\beta \frac{T_{0}}{h} \sqrt{\pi a^{\mathrm{cr}}},
$$

so that we obtain the following expression for the critical value of tension:

$$
T_{0}^{\mathrm{cr}} \equiv\left(T_{0}\right)_{a=a^{\mathrm{cr}}}=\frac{K_{\mathrm{C}} h}{\beta \sqrt{\pi a^{\mathrm{cr}}}} .
$$

Thus, safe movement of the plate is realised when

$$
T_{0} \leq T_{0}^{\mathrm{cr}} .
$$

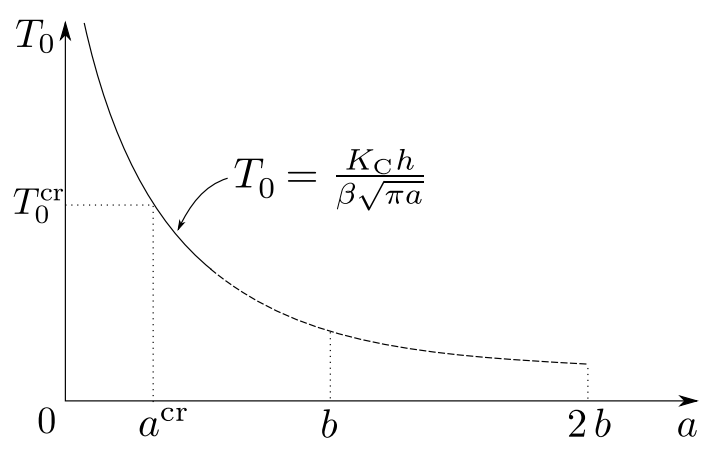

Figure 4: An admissible length of the crack and the critical tension, a schematic figure. Note the assumption $a^{\text {cr }}=a^{*} \ll 2 b$.

If the tension increases and activates the critical value (8) (see Fig. 4), the crack will propagate without stop and cut the web into two. This process of the crack growth is realised in a dynamical manner and is considered as inadmissible and catastrophic for applications.

The crack size limits the value of tension, which in turn limits the value of the critical 
velocity. We now take into account the movement of the plate. From the viewpoint of fracture, we assume that the rollers do not affect the crack behaviour.

When interested in the upper limit of the web velocity corresponding to a critical crack size (and the critical value of tension), we may use Eq. (2) where the value of tension is the critical value given in (8). In this way, the following safe range of velocities is obtained:

$$
0<V_{0}<V_{0}^{*} \equiv \sqrt{\gamma_{*} \frac{\pi^{2} D}{m \ell^{2}}+\frac{K_{\mathrm{C}} h}{\beta m \sqrt{\pi a^{\mathrm{cr}}}}} .
$$

\section{$5 \quad$ Fatigue Fracture Under Cyclic Tension}

The plate is assumed to undergo cyclic loading and unloading processes caused by cyclic variation in the value of in-plane tension $T$ (Fig. 2). For one cycle, tension increases from $T=T_{\min }$ up to $T=T_{\max }$ (the loading process) and then decreases from $T=T_{\max }$ to $T=T_{\min }$ (the unloading process). We suppose quasi-static processes meaning that the dynamic effects are excluded.

We define parameters $T_{0}$ (average tension) and $\Delta T$ (small tension variation) such that

$$
\begin{gathered}
T_{\min }=T_{0}-\Delta T \text { and } T_{\max }=T_{0}+\Delta T, \\
T_{\min } \leq T \leq T_{\max },
\end{gathered}
$$

and

$$
T_{0}-\Delta T>0 \quad \text { and } \quad \frac{\Delta T}{T_{0}} \ll 1 .
$$

We consider the plate longevity (number of loading cycles) applying the fatigue crack growth theory. Suppose that the web contains one initial crack of length $a_{0}$. The process of 
fatigue crack growth under cyclic tension (loading) can be described by the Paris' law (Paris and Erdogan, 1963). The describing equation and initial condition are

$$
\frac{\mathrm{d} a}{\mathrm{~d} n}=C(\Delta K)^{k}, \quad(a)_{n=0}=a_{0},
$$

where the variation $\Delta K$ of the stress intensity factor $K$ (defined in (6)) can be expressed as

$$
\Delta K=\frac{2 \beta \sqrt{\pi a}}{h} \Delta T .
$$

In Eq. (11), $C$ and $k$ are material constants and $n$ is the number of cycles.

The ordinary differential equation (11) defines a quasi-static process of crack growth, and determines the dependence of the crack length $a$ on the number of cycles $n$, and is valid up to the moment, when $a=a^{\text {cr }}$ and the unstable crack growth (fracture of the web) is attained.

Suppose that the unstable crack growth is attained after $n=n^{\text {cr }}$ cycles, when the critical crack length $a^{\text {cr }}$ satisfies the limiting relation

$$
\left(K_{\max }\right)_{a=a^{\text {cr }}}=\beta \frac{T_{\max }}{h} \sqrt{\pi a^{\text {cr }}}=K_{\mathrm{C}} .
$$

Note that $T_{\max }$ and $T_{\min }$ are the maximum and minimum tensions in the non-cracked web at the crack location. Thus, the structural longevity can be measured by the number of load cycles

$$
n=n^{\mathrm{cr}},
$$

for which $a=a^{\text {cr }}$, and the unstable fracture is realised. In the analysis process, the longevity constraint can be taken as

$$
n^{\mathrm{cr}} \geq n_{\mathrm{C}},
$$


where $n_{\mathrm{C}}$ is a given minimum value of cycles.

Using (11) and (12), we write the crack growth equation in the following form:

$$
\frac{\mathrm{d} a}{\mathrm{~d} n}=C \kappa_{0}^{k} a^{k / 2}, \quad \kappa_{0}=\frac{2 \beta \sqrt{\pi}}{h} \Delta T .
$$

It follows from (16) and the initial condition in (11) that for considered values of the parameter $k \neq 2$, we will have

$$
n=A\left[\frac{1}{a_{0}^{(k-2) / 2}}-\frac{1}{a^{(k-2) / 2}}\right], \quad A=\frac{2}{(k-2) C \kappa_{0}^{k}} .
$$

Using (13) and the inequality $\Delta T / T_{0} \ll 1$, we obtain

$$
a^{\mathrm{cr}}=\frac{1}{\pi}\left(\frac{K_{\mathrm{C}} h}{\beta T_{\max }}\right)^{2} \approx \frac{1}{\pi}\left(\frac{K_{\mathrm{C}} h}{\beta T_{0}}\right)^{2},
$$

and, consequently, we will have the following expression for the critical number of cycles:

$$
n^{\mathrm{cr}}=(n)_{a=a^{\mathrm{cr}}}=A\left[\frac{1}{a_{0}^{(k-2) / 2}}-\left(\frac{\sqrt{\pi} \beta T_{0}}{K_{\mathrm{C}} h}\right)^{k-2}\right] .
$$

From the condition of positiveness of the expression in (19), we find the maximum value of admissible tensions

$$
T_{0} \leq \frac{1}{\sqrt{\pi a_{0}}} \frac{K_{\mathrm{C}} h}{\beta} \equiv T_{0}^{\mathrm{M}} .
$$

In the special case $k=2$, we can find the critical number of cycles to be

$$
n^{\mathrm{cr}}=B \ln \left[\frac{1}{\pi a_{0}}\left(\frac{K_{\mathrm{C}} h}{\beta T_{0}}\right)^{2}\right], \quad B=\frac{1}{C \kappa_{0}^{2}}, \quad T_{0} \leq T_{0}^{\mathrm{M}},
$$


and the tension limit $T_{0}^{\mathrm{M}}$ is expressed by $(20)$.

The dependence of the critical number of cycles $n^{\mathrm{cr}}$ on the average tension $T_{0}$ and the problem parameter $k$ is shown in Fig. 5 using dimensionless quantities defined below in (26)-(28).

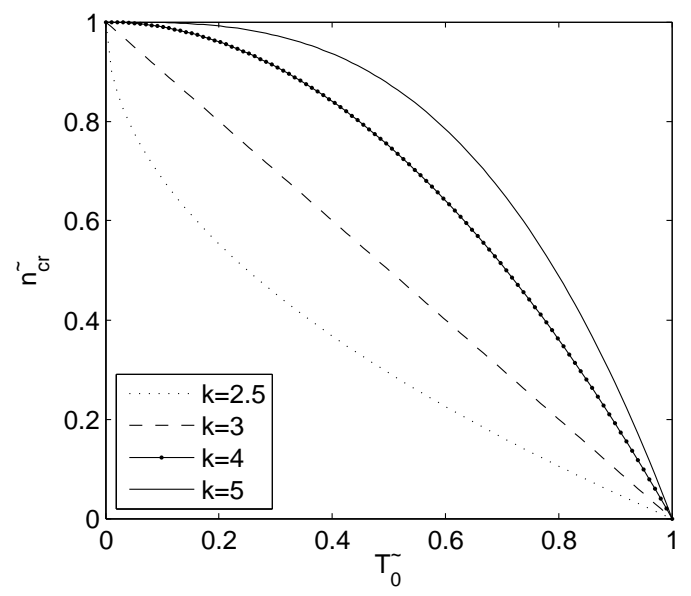

Figure 5: Dependence of the critical number of cycles (dimensionless) on the average tension (dimensionless).

The critical value of tension maximum $T_{\max }^{\mathrm{cr}}$ (or approximation of average $T_{0}^{\mathrm{cr}}$ ) may be studied with respect to the problem parameters. For example, if we set $n^{\text {cr }}=n_{\mathrm{C}}$, we may solve $T_{\max }^{\mathrm{cr}}$ from (18)-(19) (or from (18) and (21) if $k=2$ ). 
Optimization of Processes with Moving Materials

\section{Optimal Value for In-Plane Tension via Multi-Criteria Optimization}

We present a productivity criterion (performance function) with the help of the plate velocity $V_{0}$ and the process time $t_{\mathrm{f}}$ :

$$
M=m_{0} V_{0} t_{\mathrm{f}}, \quad m_{0}=2 b m .
$$

where

$$
\begin{gathered}
0<V_{0}<V_{0}^{\text {cr }}, \\
0<t_{\mathrm{f}}<t_{\mathrm{f}}^{\text {cr }} \quad \text { or } 0<n<n^{\text {cr }},
\end{gathered}
$$

and furthermore, the critical value of the velocity $V_{0}^{\text {cr }}$ is given by $(2)$, and the critical number of the cycles $n^{\text {cr }}$ by (19) (and by (21) in the case $k=2$ ). For a small cycle time period $\tau$ and a big number of cycles $n$, we assume that $t_{\mathrm{f}}=n \tau$ (approximately).

Using the critical velocity $V_{0}^{\text {cr }}$, longevity $n^{\text {cr }}$ and process effectiveness $M^{\text {cr }}$ and noticing that these values depend on the value of in-plane average tension $T_{0}$, we define the following vector function:

$$
J\left(T_{0}\right)=\left\{\begin{array}{c}
J_{\mathrm{V}}\left(T_{0}\right) \\
J_{\mathrm{N}}\left(T_{0}\right) \\
J_{\mathrm{M}}\left(T_{0}\right)
\end{array}\right\} \equiv\left\{\begin{array}{c}
V_{0}^{\mathrm{cr}}\left(T_{0}\right) \\
n^{\mathrm{cr}}\left(T_{0}\right) \\
M^{\mathrm{cr}}\left(T_{0}\right)
\end{array}\right\},
$$

where $M^{\text {cr }}$ is given by (22) with critical parameter values.

Now, we formulate the multi-objective optimization problem. It is required to determine the optimal value $T_{0}^{*}$ of in-plane tension $T_{0}$ that gives a maximum of the considered vector 
function, i.e.

$$
J^{*}=J\left(T_{0}^{*}\right)=\max _{T_{0}} J\left(T_{0}\right) .
$$

The max operation in (24) is considered in the Pareto sense. It is

$$
T_{0}^{*}=\arg \max _{T_{0}} J\left(T_{0}\right)
$$

if there is no other value $\hat{T}_{0}$, such that

$$
J_{i}\left(\hat{T}_{0}\right) \geq J_{i}\left(T_{0}^{*}\right), \quad i=\mathrm{V}, \mathrm{N}, \mathrm{M},
$$

and the following rigorous inequality is satisfied for at least one component criterion:

$$
J_{j}\left(\hat{T}_{0}\right)>J_{j}\left(T_{0}^{*}\right) .
$$

To solve this multi-objective optimization problem, we apply the weighting method, which is one of the most relevant substitutes for vector optimization problems. The preference function is formulated as a sum of the single objective functions $J_{\mathrm{V}}, J_{\mathrm{N}}, J_{\mathrm{M}}$ associated with the weighting factors $C_{\mathrm{V}}, C_{\mathrm{N}}, C_{\mathrm{M}}$ :

$$
J_{\mathrm{C}}=C_{\mathrm{V}} J_{\mathrm{V}}+C_{\mathrm{N}} J_{\mathrm{N}}+C_{\mathrm{M}} J_{\mathrm{M}},
$$

where we suppose that

$$
\begin{gathered}
C_{\mathrm{V}} \geq 0, C_{\mathrm{N}} \geq 0, C_{\mathrm{M}} \geq 0, \\
C_{\mathrm{V}}+C_{\mathrm{N}}+C_{\mathrm{M}}=1 .
\end{gathered}
$$


We will consider the multi-objective optimization problem of finding the optimal in-plane tension $T_{0}^{*}$ separately for different particular cases using the expressions presented in Section 2.

For convenience of performing the analysis and for reduction of characteristic parameters, we introduce the following values with tildes:

$$
\begin{aligned}
\tilde{J}_{\mathrm{V}} & =\frac{J_{\mathrm{V}}}{J_{\mathrm{V}}^{0}}, & J_{\mathrm{V}}^{0} & =\sqrt{\frac{K_{\mathrm{C}} h}{m \beta \sqrt{\pi a_{0}}}}, \\
\tilde{J}_{\mathrm{N}} & =\frac{J_{\mathrm{N}}}{J_{\mathrm{N}}^{0}}, & J_{\mathrm{N}}^{0} & =\frac{2}{(k-2) C \kappa_{0}^{k} a_{0}^{(k-2) / 2}}, \\
\tilde{J}_{\mathrm{M}} & =\frac{J_{\mathrm{M}}}{J_{\mathrm{M}}^{0}}, & J_{\mathrm{M}}^{0} & =m_{0} \tau J_{\mathrm{V}}^{0} J_{\mathrm{N}}^{0},
\end{aligned}
$$

and represent the criterion functions as

$$
\begin{aligned}
& \tilde{J}_{\mathrm{V}}=\left(\tilde{T}_{0}+d\right)^{1 / 2}, \\
& \tilde{J}_{\mathrm{N}}=1-\tilde{T}_{0}^{k-2}, \\
& \tilde{J}_{\mathrm{M}}=\tilde{J}_{\mathrm{V}} \tilde{J}_{\mathrm{N}}
\end{aligned}
$$

using the dimensionless values of problem parameters

$$
\tilde{T}_{0}=\frac{\beta \sqrt{\pi a_{0}}}{K_{\mathrm{C}} h} T_{0}, \quad d=\frac{\gamma_{*}^{2} \pi^{2} D \beta \sqrt{\pi a_{0}}}{l^{2} K_{\mathrm{C}} h}, \quad 0<\tilde{T}_{0}<1
$$




\subsection{Maximizing the Critical Velocity and Safety Criterion}

We consider the case of maximization of the velocity criterion $\tilde{J}_{\mathrm{V}}$ and the safety criterion $\tilde{J}_{\mathrm{N}}$ when $k=3$. In this case, we have

$$
\begin{gathered}
\tilde{J}_{1} \equiv C_{\mathrm{V}} \tilde{J}_{\mathrm{V}}+C_{\mathrm{N}} \tilde{J}_{\mathrm{N}}, \\
C_{\mathrm{V}}+C_{\mathrm{N}}=1 .
\end{gathered}
$$

Let us study the solution of (29) with respect to the weight $C_{\mathrm{N}}$. Now, the optimization problem is $\left(C_{\mathrm{V}}=1-C_{\mathrm{N}}\right)$

$$
\max _{0 \leq \tilde{T}_{0} \leq 1}\left(1-C_{\mathrm{N}}\right)\left(\tilde{T}_{0}+d\right)^{1 / 2}+C_{\mathrm{N}}\left(1-\tilde{T}_{0}\right) .
$$

The object function in (30) is concave, so the weighting method may be used for finding the Pareto optimal solutions.

Since the object function is concave, the solution $\tilde{T}_{0}^{*}$ of $(30)$ can be found at a zero of the derivative of the object function if it lies on the interval $[0,1]$. Otherwise, the solution is 0 or 1 . The solution $\tilde{T}_{0}^{*}$ depends on the weight $C_{\mathrm{N}}$ and it can be found analytically. The following dependence of the optimal value for the dimensionless tension $\tilde{T}_{0}^{*}$ on $C_{\mathrm{N}}$ holds:

$$
\begin{array}{rlrl}
0 & \leq C_{\mathrm{N}} \leq \frac{1}{1+2 \sqrt{d+1}}: & & \tilde{T}_{0}^{*}=1, \\
\frac{1}{1+2 \sqrt{d+1}}<C_{\mathrm{N}}<\frac{1}{1+2 \sqrt{d}}: & & \tilde{T}_{0}^{*}=\left(\frac{1-C_{\mathrm{N}}}{2 C_{\mathrm{N}}}\right)^{2}-d, \\
\frac{1}{1+2 \sqrt{d}} & \leq C_{\mathrm{N}} \leq 1: & & \tilde{T}_{0}^{*}=0 .
\end{array}
$$


Consequently, the values of the component functions are found in the following form:

$$
\begin{aligned}
& \tilde{J}_{\mathrm{V}}=\sqrt{\tilde{T}_{0}^{*}+d}=\frac{1-C_{\mathrm{N}}}{2 C_{\mathrm{N}}} \\
& \tilde{J}_{\mathrm{N}}=1-\tilde{T}_{0}^{*}=1+d-\left(\frac{1-C_{\mathrm{N}}}{2 C_{\mathrm{N}}}\right)^{2}
\end{aligned}
$$

and, for the considered problem of critical velocity and longevity maximization, the Pareto front $(\mathrm{PF})$ of the optimal solution is given by the equation

$$
\tilde{J}_{\mathrm{N}}=1+d-\tilde{J}_{\mathrm{V}}^{2}
$$

where $\tilde{J}_{\mathrm{V}} \in[\sqrt{d}, \sqrt{1+d}]$.

\subsection{Maximization of the Critical Velocity and Process Effective- ness}

Consider now another case, where we maximize the functions $\tilde{J}_{\mathrm{V}}$ (critical velocity criterion)

and $\tilde{J}_{\mathrm{M}}$ (process effectiveness criterion). We discuss again the case $k=3$. In this case, the weighting method problem is

$$
\begin{gathered}
\tilde{J}_{2} \equiv C_{\mathrm{V}} \tilde{J}_{\mathrm{V}}+C_{\mathrm{M}} \tilde{J}_{\mathrm{M}}, \\
C_{\mathrm{V}}+C_{\mathrm{M}}=1,
\end{gathered}
$$

so that we study

$$
\max _{0 \leq \tilde{T}_{0} \leq 1} C_{\mathrm{V}}\left(\tilde{T}_{0}+d\right)^{1 / 2}+C_{\mathrm{M}}\left(\tilde{T}_{0}+d\right)^{1 / 2}\left(1-\tilde{T}_{0}\right)
$$


The object function in $(32)$ is concave. Now, the extremal condition is

$$
\begin{aligned}
\frac{\mathrm{d} \tilde{J}_{2}}{\mathrm{~d} \tilde{T}_{0}} & =C_{\mathrm{V}} \frac{\mathrm{d} \tilde{J}_{\mathrm{V}}}{\mathrm{d} \tilde{T}_{0}}+C_{\mathrm{M}} \frac{\mathrm{d} \frac{\tilde{J}_{\mathrm{M}}}{\mathrm{d} \tilde{T}_{0}}}{} \\
& =C_{\mathrm{V}} \frac{\mathrm{d} \tilde{J}_{\mathrm{V}}}{\mathrm{d} \tilde{T}_{0}}+C_{\mathrm{M}}\left(\tilde{J}_{\mathrm{N}} \frac{\mathrm{d} \tilde{J}_{\mathrm{V}}}{\mathrm{d} \tilde{T}_{0}}+\tilde{J}_{\mathrm{V}} \frac{\mathrm{d} \tilde{J}_{\mathrm{N}}}{\mathrm{d} \tilde{T}_{0}}\right) \\
& =0
\end{aligned}
$$

The solution of the problem is studied with respect to the weight $C_{\mathrm{M}}$. By (33), it is found that the optimal value for the dimensionless tension $\tilde{T}_{0}^{*}$ depends on $C_{\mathrm{M}}$ as follows:

$$
\begin{array}{rlrl}
0 & \leq C_{\mathrm{M}} \leq \frac{1}{2 d+3}: & \tilde{T}_{0}^{*}=1 \\
\frac{1}{2 d+3}<C_{\mathrm{M}} \leq 1: & \tilde{T}_{0}^{*}=\frac{1-2 d C_{\mathrm{M}}}{3 C_{\mathrm{M}}} .
\end{array}
$$

For the optimized functions $\tilde{J}_{\mathrm{V}}$ and $\tilde{J}_{\mathrm{M}}$, we have

$$
\begin{aligned}
& \tilde{J}_{\mathrm{V}}^{2}=\frac{1}{3}\left(\frac{1}{C_{\mathrm{M}}}+d\right) \\
& \tilde{J}_{\mathrm{M}}=\frac{1}{3}\left(2 d+3-\frac{1}{C_{\mathrm{M}}}\right) \sqrt{\frac{1}{3}\left(\frac{1}{C_{\mathrm{M}}}+d\right)}
\end{aligned}
$$

The Pareto front $(\mathrm{PF})$ of the problem under consideration is described by the equation

$$
\tilde{J}_{\mathrm{M}}=(1+d) \tilde{J}_{\mathrm{V}}-\tilde{J}_{\mathrm{V}}^{3}
$$

defined on the interval

$$
\tilde{J}_{\mathrm{V}} \in[\sqrt{(1+d) / 3}, \sqrt{1+d}] .
$$




\subsection{Maximizing the Safety Criterion and Process Effectiveness}

As a third case, we study the maximization of the functions $\tilde{J}_{\mathrm{N}}$ (safety criterion) and $\tilde{J}_{\mathrm{M}}$ (process effectiveness criterion) when $k=3$. We have

$$
\begin{gathered}
\tilde{J}_{3} \equiv C_{\mathrm{N}} \tilde{J}_{\mathrm{N}}+C_{\mathrm{M}} \tilde{J}_{\mathrm{M}}, \\
C_{\mathrm{N}}+C_{\mathrm{M}}=1,
\end{gathered}
$$

and the optimization problem reads

$$
\max _{0 \leq \tilde{T}_{0} \leq 1} C_{\mathrm{N}}\left(1-\tilde{T}_{0}\right)+\left(1-C_{\mathrm{N}}\right)\left(\tilde{T}_{0}+d\right)^{1 / 2}\left(1-\tilde{T}_{0}\right)
$$

The object function $\tilde{J}_{3}$ is concave. We study the problem (35) with respect to the weight $C_{\mathrm{N}}$. Now the optimal value of the dimensionless tension $\tilde{T}_{0}^{*}$ depends on $C_{\mathrm{N}}$ in the following way:

$$
\begin{array}{ll}
0 \leq C_{\mathrm{N}}<\frac{1-2 d}{1-2 d+2 \sqrt{d}}: & \tilde{T}_{0}^{*}=\frac{2}{9}\left(\alpha^{2}-3 d+3 / 2-\alpha \sqrt{\alpha^{2}+3 d+3}\right), \\
\frac{1-2 d}{1-2 d+2 \sqrt{d}} \leq C_{\mathrm{N}} \leq 1: & \tilde{T}_{0}^{*}=0,
\end{array}
$$

where

$$
\alpha=C_{\mathrm{N}} / C_{\mathrm{M}}=C_{\mathrm{N}} /\left(1-C_{\mathrm{N}}\right) .
$$

In this case, the Pareto front is given by

$$
\tilde{J}_{\mathrm{M}}=\tilde{J}_{\mathrm{N}} \sqrt{1+d-\tilde{J}_{\mathrm{N}}}, \quad \tilde{J}_{\mathrm{N}} \in\left[\frac{2}{3}(1+d), 1\right] .
$$


Note that the maximum of (25) in the case $C_{\mathrm{M}}=1$ and $C_{\mathrm{V}}=C_{\mathrm{N}}=0$ is found above by solving the problems (32) and (35). Then, $\tilde{J}_{\mathrm{V}}=\sqrt{(1+d) / 3}$ and $\tilde{J}_{\mathrm{N}}=\frac{2}{3}(1+d)$, which is also a Pareto optimal solution for the problem (30) confirmed by (31).

\section{$7 \quad$ Numerical Results and Discussion}

The results obtained in previous sections are illustrated numerically in this section. Parameter values are chosen such that they describe paper materials and paper making process conditions. They are $\nu=0.3, E=10^{9} \mathrm{~Pa}, m=0.08 \mathrm{~kg} / \mathrm{m}^{2}, h=10^{-4} \mathrm{~m}, \ell=0.1 \mathrm{~m}$, $2 b=10 \mathrm{~m}$, and $\beta=1.12$. Paper material properties have been measured by, e.g., Seth and Page (1974), and Yokoyama and Nakai (2007).

We first study the case with constant tension. In Fig. 6(a), the predicted critical value

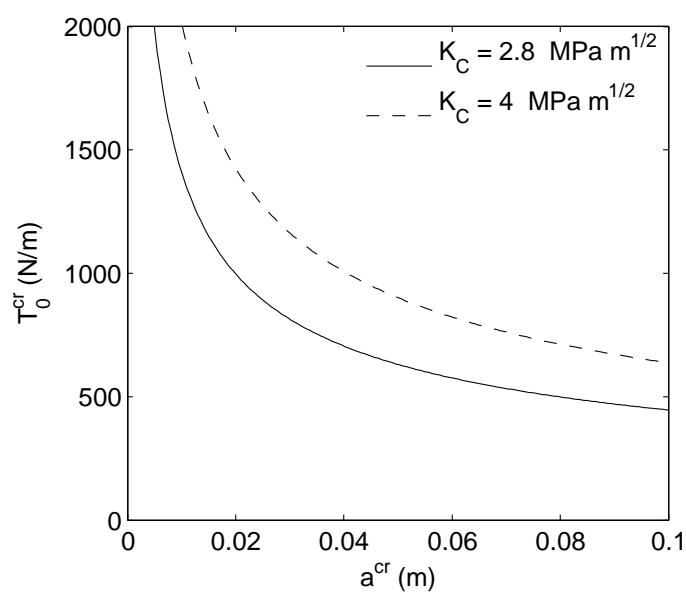

(a)

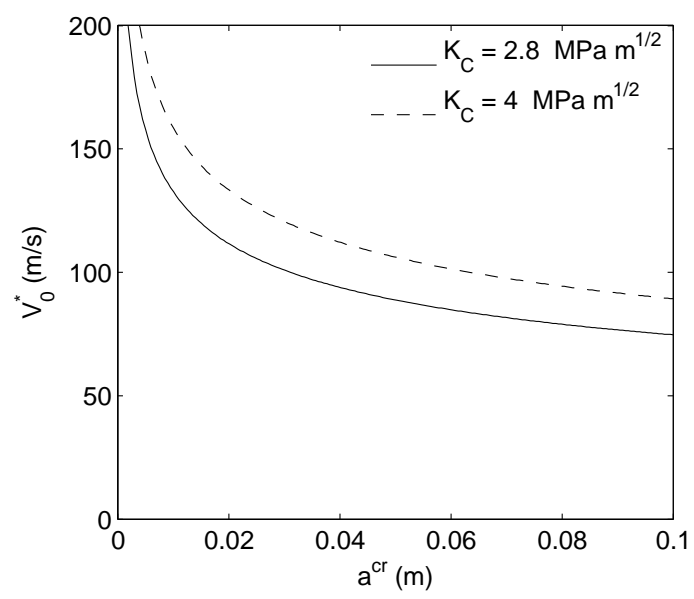

(b)

Figure 6: Critical tension (a) and the corresponding critical velocity (b) with respect to the critical crack length for two different values of the strain energy rate.

of tension $T_{0}^{\mathrm{cr}}$ is plotted with respect to the critical crack length $a^{\mathrm{cr}}(\mathrm{Eq} .(8))$. The results are plotted for two different values of $K_{\mathrm{C}}$ : the smaller one $\left(2.8 \cdot 10^{6} \mathrm{~Pa} \sqrt{\mathrm{m}}\right)$ corresponding to 
a newsprint and the larger one $\left(4 \cdot 10^{6} \mathrm{~Pa} \sqrt{\mathrm{m}}\right)$ corresponding to a writing paper (Seth and Page, 1974). The results are plotted for the range $0 \leq a^{\mathrm{cr}} \leq 0.1 \mathrm{~m}$.

In Fig. 6(b), the upper limit of safe velocity $V_{0}^{*}$ (in vacuum) is plotted with respect to the critical crack length $a^{\text {cr }}$ (Eq. (10)). The results suggest that a safe web velocity is higher for a web with smaller initial cracks.

Note that we assume $a^{\text {cr }} \ll 2 b$, which allows us to use a constant crack geometry factor $\beta=1.12$. The results in Fig. 6 are independent from the parameter $b$ but the accuracy of the results depends on $b$. Thus, the results in Fig. 6 may not be valid for very narrow webs $(2 b=0.1 \mathrm{~m})$.

We then study fatigue fracture of the travelling plate under cyclic tension. In Fig. 7(b), the value of critical tension maximum $T_{\max }^{\mathrm{cr}}$ is studied as a function of problem parameters $a_{0}$ and $\Delta T$. In Fig. $7($ a), the values of the critical crack length corresponding to the values of critical tension are shown. We set the value for the number of cycles the web must sustain to be $n_{\mathrm{C}}=100$. In Paris' law, we used values $k=3$ and $C=10^{-18}$. Paris' constants have been measured for many materials, e.g. for nickel based superalloys by Bazant and $\mathrm{Xu}$ (1991), for concrete by Branco et al. (2009), and for epoxy by Brown et al. (2009), and the constants vary a lot for different materials. Parameter $k$ is approximately 3 for many materials (Farahmand and Nikbin, 2008) but it may also be larger (for epoxy, $k=9.7$, see Brown et al., 2009) or smaller (for rubber, $k=0.211$, see Schubel et al., 2003). Values for parameter $C$ vary between $10^{-40}$ and 1 .

In Fig. 7, the used value for fracture toughness was $K_{\mathrm{C}}=2.8 \cdot 10^{6} \mathrm{~Pa} \sqrt{\mathrm{m}}$. The initial crack length $a_{0}$ was given values $0.01 \mathrm{~m}-0.1 \mathrm{~m}$, and the variation in tension was between $0.1 \mathrm{~N} / \mathrm{m}$ and $5 \mathrm{~N} / \mathrm{m}$. The critical number of cycles was constant, $n_{\mathrm{C}}=n^{\mathrm{cr}}=100$. The results are as expected. If the variation in tension is big, only small initial cracks can be 


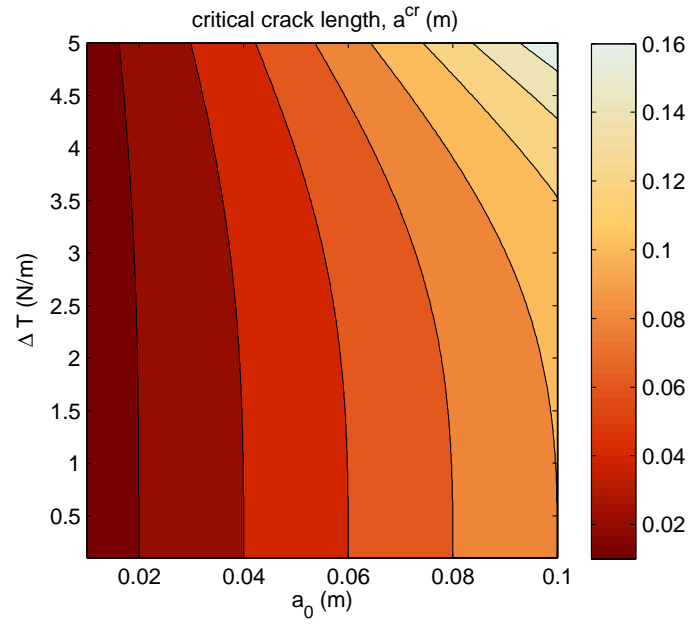

(a)

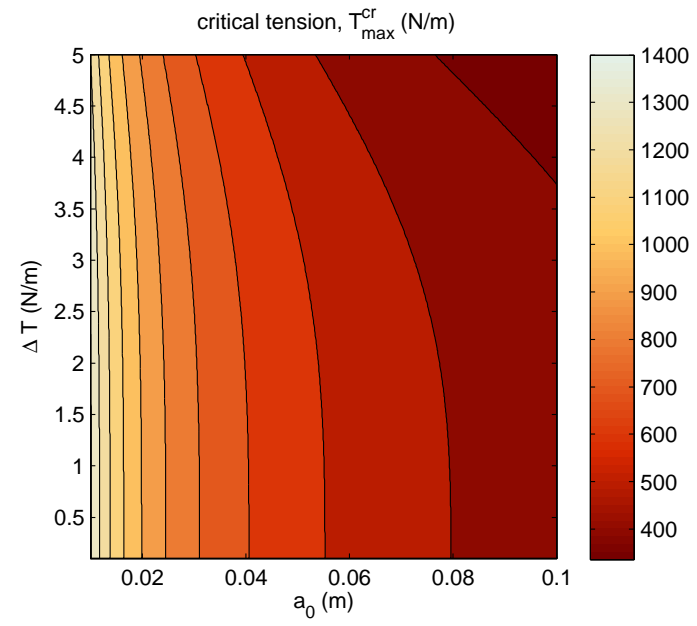

(b)

Figure 7: Critical crack length $a^{\mathrm{cr}}$ (a) and critical tension $T_{\max }^{\mathrm{cr}}$ (b) with respect to the initial crack length $a_{0}$ and the tension variation $\Delta T$. The number of cycles $n^{\text {cr }}=n_{\mathrm{C}}=100$ is fixed.

accepted. Note that in the figure, the critical conditions are shown. Also the number of cycles corresponds to the critical situation. These results are mainly qualitative.

Finally, we analyse the results of the optimization problems. The Pareto fronts (31), (34) and (36) are illustrated in Fig. 8 when the initial crack length is $a_{0}=0.01 \mathrm{~m}$.
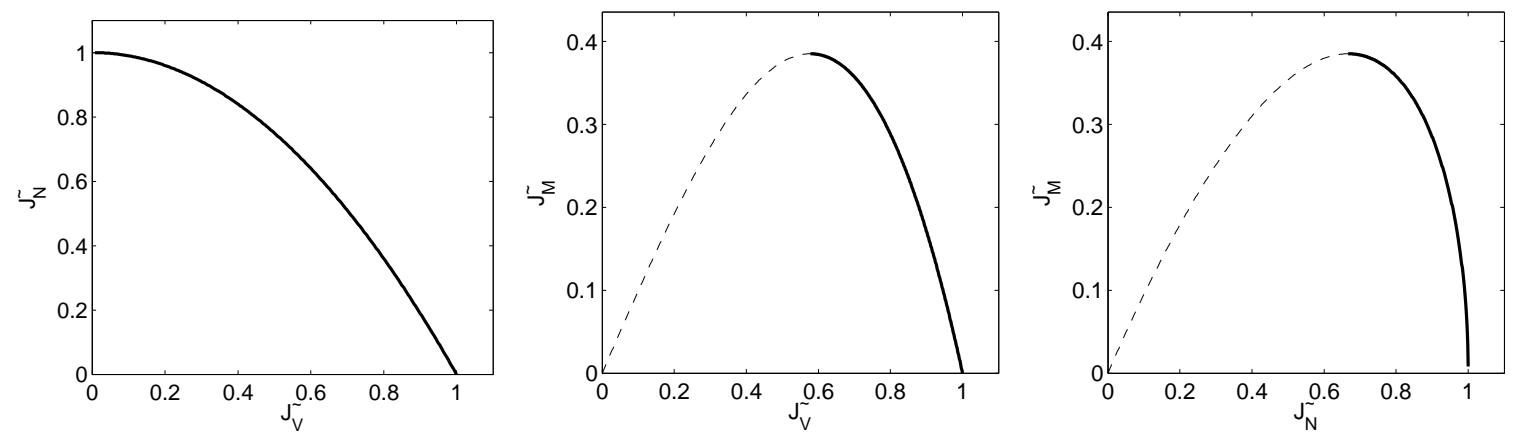

Figure 8: Pareto fronts for the problems $\max \left\{\tilde{J}_{\mathrm{N}}, \tilde{J}_{\mathrm{V}}\right\}, \max \left\{\tilde{J}_{\mathrm{M}}, \tilde{J}_{\mathrm{V}}\right\}$, and $\max \left\{\tilde{J}_{\mathrm{M}}, \tilde{J}_{\mathrm{N}}\right\}$, respectively, in the case when the initial crack length $a_{0}=0.01 \mathrm{~m}$.

In Fig. 9, the optimal values of tension $T_{0}^{*}(\mathrm{~N} / \mathrm{m})$ for the problems $(30),(32)$ and (35) 
are plotted with respect to the weights $\left(C_{\mathrm{N}}, C_{\mathrm{M}}\right.$ and $C_{\mathrm{N}}$, respectively $)$ and the initial length of the crack $a_{0}$.

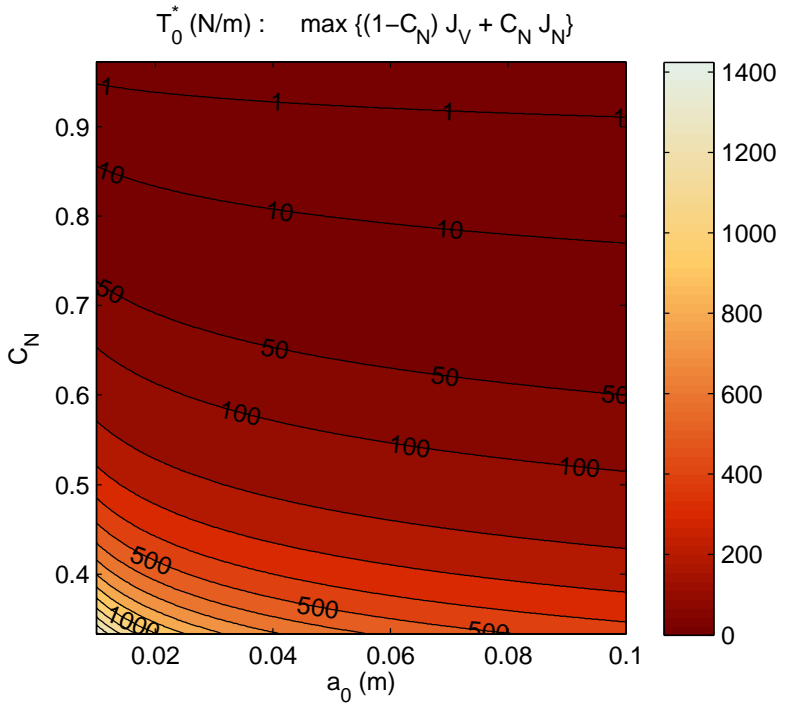

(a)

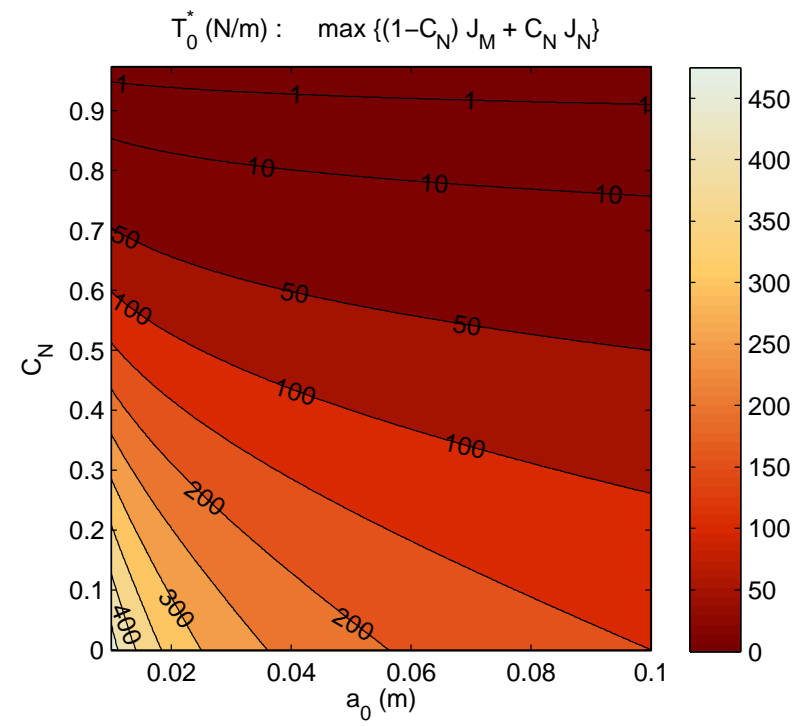

(c)

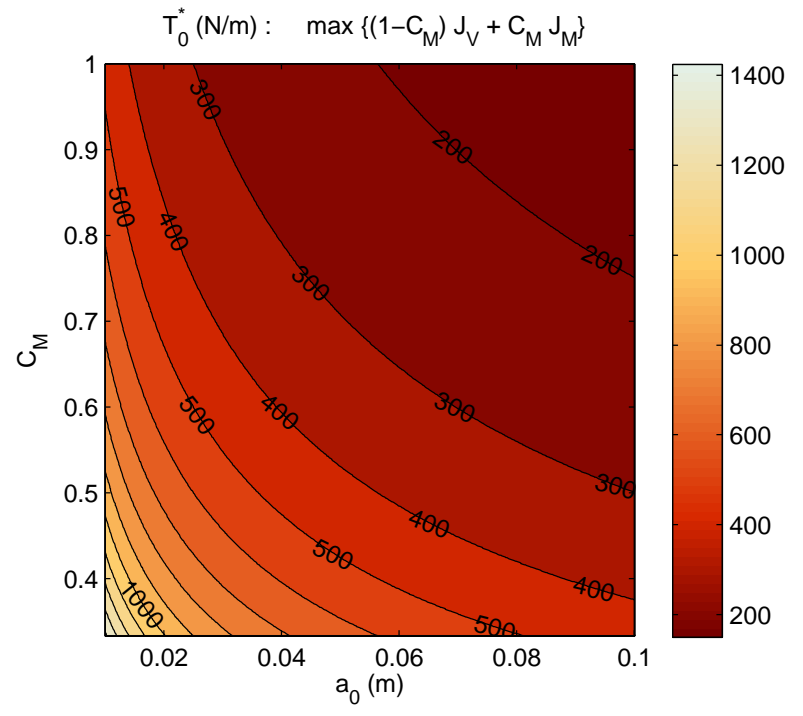

(b)

Figure 9: Dependence of the optimal tension $T_{0}$ on the parameter $a_{0}$ and the weight $C_{\mathrm{N}}$ or $C_{\mathrm{M}}$.

In Fig. 9(a), we present the optimal values of tension $T_{0}^{*}$ when the velocity $J_{\mathrm{V}}$ is optimized (weighted) against the longevity $J_{N}$. One may note that even for a small crack size $\left(a_{0}=\right.$ 
0.01), the optimal value of tension is almost zero, when the longevity is given a large weight $\left(C_{N}>0.8\right)$. Weighting the velocity, the optimal tension gets very large values $\left(T_{0}^{*} \sim 1400\right.$ $\mathrm{N} / \mathrm{m})$.

In Fig. 9(b), we weight the velocity function $J_{\mathrm{V}}$ against the process effectiveness function $J_{\mathrm{M}}$. In this case, it is noted that the length of the initial crack length significantly affects the optimal value of tension.

Figure $9(\mathrm{c})$ shows the third case, where the longevity $J_{\mathrm{N}}$ and the process effectiveness $J_{\mathrm{M}}$ are compared. Also here, it is seen that $a_{0}$ has an effect on the value of optimal tension, especially when the process effectiveness is weighted.

Table 1: Dependence of the optimal tension (dimensionless $\tilde{T}_{0}^{*}$ and dimensional $T_{0}^{*}$ ) on the selected weights for the three studied cases. The used initial crack length was $a_{0}=0.01 \mathrm{~m}$.

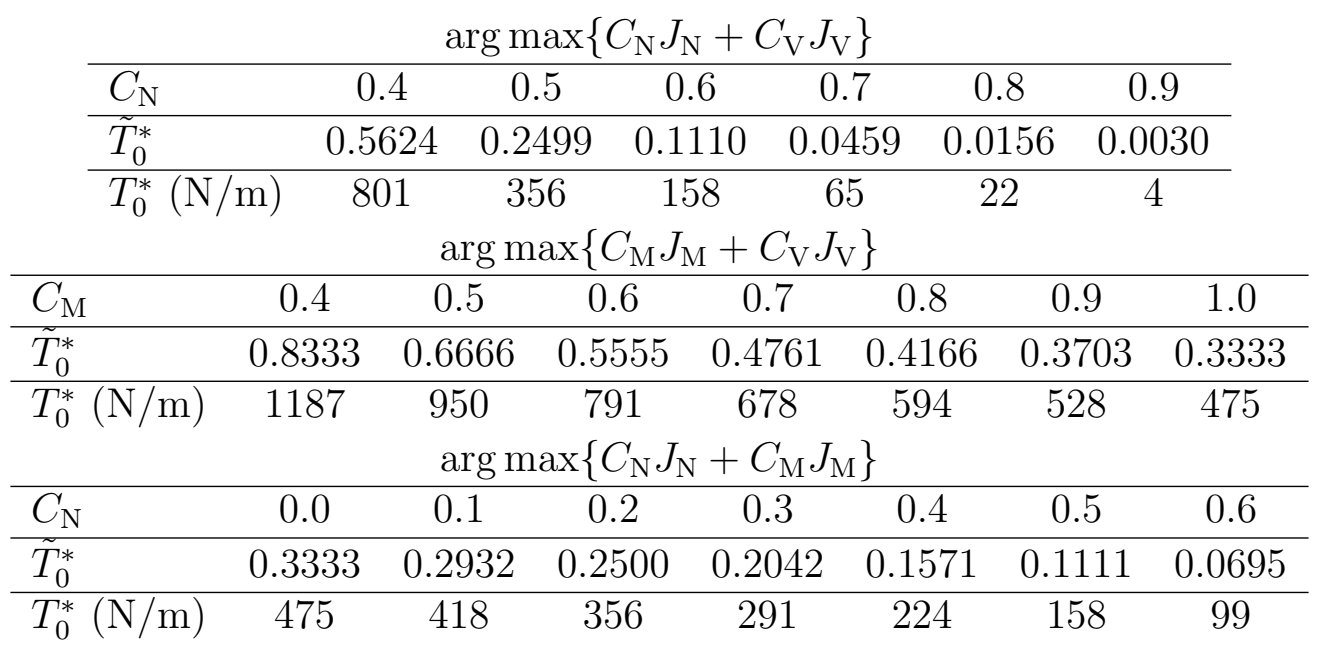

Note that the case $C_{\mathrm{M}}=1$ and $C_{\mathrm{V}}=C_{\mathrm{N}}=0$ is included in both Figs. $9(\mathrm{~b})$ and $9(\mathrm{c})$, being the worst case in the previous figure and the best one in the latter figure. Analysing these two sub-problems helps us to make decisions on the weights to be selected. The optimum of process effectiveness $J_{\mathrm{M}}$ gives some kind of reference value for the desired tension.

With the help of Fig. 9, some values of the weights $C_{\mathrm{N}}$ and $C_{\mathrm{M}}$ were selected and the 
solutions were collected and are shown in Table 1 . The initial crack length $a_{0}$ gets values $0.005 \mathrm{~m}, 0.01 \mathrm{~m}, 0.05 \mathrm{~m}$ and $0.1 \mathrm{~m}$. In Table 1 , also the dimensional values $(\mathrm{N} / \mathrm{m})$ for tension are shown. In the case $C_{\mathrm{M}}=1, C_{\mathrm{V}}=C_{\mathrm{N}}=0$, the optimal tension level of the process is $\tilde{T}_{0}^{*}=0.3333$ or $T_{0}^{*}=475 \mathrm{~N} / \mathrm{m}$. Comparing this to the results for a stationary paper web (Eq. (18) and Fig. 6(a)), we see that tension level $T_{0}=475 \mathrm{~N} / \mathrm{m}$ corresponds to the critical crack of length $a_{\mathrm{cr}}=0.088 \mathrm{~m}$ for $K_{\mathrm{C}}=2.8 \cdot 10^{6} \mathrm{~Pa} \sqrt{\mathrm{m}}$ and $a_{\mathrm{cr}}=0.18 \mathrm{~m}$ for $K_{\mathrm{C}}=4 \cdot 10^{6} \mathrm{~Pa} \sqrt{\mathrm{m}}$.

\section{Conclusions}

This paper was devoted to finding optimal conditions in a system, where material (continuum) travels between a system of supports (rollers). The model of a thin elastic plate subject to bending, in-plane tension and fatigue crack growth was used. The optimized criteria were maximal critical web speed, maximal structural longevity and maximal process effectiveness. Several sub-problems of the derived multi-objective optimization problem were solved analytically using the weighting method to find the Pareto optimal results. The value of optimal tension at the edges of the moving web was sought in the studied cases.

The key result of this study was finding the analytical expression for process effectiveness (productivity). With the help of it, we were able to explore the whole solution set and to proceed detailed parametric studies. It also provided efficient computational analysis.

The analytically solved Pareto fronts and optimal values for tension were analysed with the help of figures and numerical tables. It was seen that the length of the initial crack affects the optimal value of tension as expected: the larger the crack size, the smaller the safe value of tension. It was noticed that the different kinds of criteria of high velocity and high 
longevity were difficult to compare to each other, but the criterion of process effectiveness could be seen as some kind of reference value to the optimal tension.

The presented way of optimizing process effectiveness, and at the same time minimizing probability of failures, in this kind of systems is new. It provides tools that can be utilized in processes with moving materials, such as paper making. However, one must note that the model used in this study was simplified: for example, the web-air interaction was excluded. The effect of the surrounding fluid is known to lower the critical speed (see e.g. Banichuk et al., 2010b).

\section{Acknowledgements}

This research was supported by the Academy of Finland (grant no. 140221) and the Jenny and Antti Wihuri Foundation.

\section{References}

Archibald, F. R. and Emslie, A. G. (1958). The vibration of a string having a uniform motion along its length. ASME Journal of Applied Mechanics, 25:347-348.

Bachene, M., Tiberkak, R., and Rechak, S. (2009). Vibration analysis of cracked plates using the extended finite element method. Archive of Applied Mechanics, 79(3):249-262.

Banichuk, N., Jeronen, J., Kurki, M., Neittaanmäki, P., Saksa, T., and Tuovinen, T. (2011a). On the limit velocity and buckling phenomena of axially moving orthotropic membranes and plates. International Journal of Solids and Structures, 48(13):2015-2025. 
Banichuk, N., Jeronen, J., Neittaanmäki, P., and Tuovinen, T. (2010a). On the instability of an axially moving elastic plate. International Journal of Solids and Structures, 47(1):9199.

Banichuk, N., Jeronen, J., Neittaanmäki, P., and Tuovinen, T. (2010b). Static instability analysis for travelling membranes and plates interacting with axially moving ideal fluid. Journal of Fluids and Structures, 26(2):274-291.

Banichuk, N., Jeronen, J., Saksa, T., and Tuovinen, T. (2011b). Static instability analysis of an elastic band travelling in the gravitational field. Rakenteiden mekaniikka (Journal of Structural Mechanics), 44(3):172-185.

Banichuk, N., Ragnedda, F., and Serra, M. (2003). Probabilistic-guaranteed optimal design of membrane shells against quasi-brittle fracture. Mechanics Based Design of Structures and Machines: An International Journal, 31(4):459-474.

Bazant, Z. P. and Xu, K. (1991). Size effect in fatigue fracture of concrete. ACI Materials Journal, 88(4):390-399.

Branco, R., Antunes, F. V., Ferreira, J. A. M., and Silva, J. M. (2009). Determination of Paris law constants with a reverse engineering technique. Engineering Failure Analysis, $16(2): 631-638$.

Brighenti, R. (2005). Numerical buckling analysis of compressed or tensioned cracked thin plates. Engineering Structures, 27(2):265-276.

Brown, E. N., White, S. R., and Sottos, N. R. (2009). Fatigue crack propagation in microcapsule-toughened epoxy. Journal of Materials Science, 41(19):6266-6273. 
Chankong, V. and Haimes, Y. Y. (1983). Multiobjective Decision Making Theory and Methodology. Elsevier Science Publishing Co., Inc., New York.

Dimarogonas, A. D. (1996). Vibration of cracked structures: A state of the art review. Engineering Fracture Mechanics, 55(5):831-857.

Farahmand, B. and Nikbin, K. (2008). Predicting fracture and fatigue crack growth properties using tensile properties. Engineering Fracture Mechanics, 75(8):2144-2155.

Garziera, R. and Amabili, M. (2000). Damping effect of winding on the lateral vibrations of axially moving tapes. ASME Journal of Vibration and Acoustics, 122:49-53.

Griffith, A. A. (1921). The phenomena of rupture and flow in solids. Philosophical Transactions of the Royal Society of London, A 221:163-198.

Hamad, W. Y. (1997). Some microrheological aspects of wood-pulp fibres subjected to fatigue loading. Cellulose, 4(1):51-56.

Hamad, W. Y. (1998). On the mechanisms of cumulative damage and fracture in native cellulose fibres. Journal of Materials Science Letters, 17(5):433-436.

Hristopulos, D. T. and Uesaka, T. (2002). A model of machine-direction tension variations in paper webs with runnability applications. Journal of Pulp and Paper Science, 28(12):389394.

Inglis, C. E. (1913). Stresses in a plate due to the presence of cracks and sharp corners. Trans. Institute of Naval Architecture, 55:219-241.

Irwin, G. R. (1958). Fracture. In Flugge, S., editor, Handbuch der Physik, volume VI, pages 551-590. Springer-Verlag, Berlin. 
Liew, K. M., Hung, K. C., and Lim, M. K. (1994). A solution method for analysis of cracked plates under vibration. Engineering Fracture Mechanics, 48(3):393-404.

Lin, C. C. (1997). Stability and vibration characteristics of axially moving plates. International Journal of Solids and Structures, 34(24):3179-3190.

Lin, C. C. and Mote, C. D. (1995). Equilibrium displacement and stress distribution in a two-dimensional, axially moving web under transverse loading. ASME Journal of Applied Mechanics, 62:772-779.

Lin, C. C. and Mote, C. D. (1996). Eigenvalue solutions predicting the wrinkling of rectangular webs under non-linearly distributed edge loading. Journal of Sound and Vibration, 197(2):179-189.

Marynowski, K. (2010). Free vibration analysis of the axially moving levy-type viscoelastic plate. European Journal of Mechanics - A/Solids, 29(5):879 - 886. DOI: 10.1016/j.euromechsol.2010.03.010.

Miettinen, K. M. (1999). Nonlinear Multiobjective Optimization, volume 12 of Kluwer's International Series in Operations Research 83 Management Science. Kluwer Academic Publishers, Boston.

Mote, C. D. (1972). Dynamic stability of axially moving materials. Shock and Vibration Digest, 4(4):2-11.

Murphy, K. D. and Zhang, Y. (2000). Vibration and stability of a cracked translating beam. Journal of Sound and Vibration, 237(2):319-335. 
Paris, P. C. and Erdogan, F. (1963). A critical analysis of crack propagation laws. Journal of Basic Engineering, Transactions of the American Society of Mechanical Engineers, D $85: 528-534$.

Prabhakara, D. L. and Datta, P. K. (1993). Vibration and static stability characteristics of rectangular plates with a localized flaw. Computers \& Structures, 49(5):825-836.

Prabhakara, D. L. and Datta, P. K. (1997). Vibration, buckling and parametric instability behaviour of plates with centrally located cutouts subjected to in-plane edge loading (tension or compression). Thin-Walled Structures, 27(4):287-310.

Schubel, P. M., Gdoutos, E. E., and Daniel, I. M. (2003). Fatigue characterization of tire rubber. In 2003 SEM Annual Conference $\&$ Exposition on Experimental and Applied Mechanics, Charlotte, North Carolina.

Seth, R. S. and Page, D. H. (1974). Fracture resistance of paper. Journal of Materials Science, 9(11):1745-1753.

Shin, C., Chung, J., and Kim, W. (2005). Dynamic characteristics of the out-of-plane vibration for an axially moving membrane. Journal of Sound and Vibration, 286(4-5):10191031.

Simpson, A. (1973). Transverse modes and frequencies of beams translating between fixed end supports. Journal of Mechanical Engineering Science, 15:159-164.

Stadler, W. (1979). A survey of multicriteria optimization or the vector maximum problem, part I: 1776-1960. Journal of Optimization Theory and Applications, 29(1):1-52. 
Stahl, B. and Keer, L. M. (1972). Vibration and stability of cracked rectangular plates. International Journal of Solids and Structures, 8(1):69-91.

Steuer, R. E. (1986). Multiple Criteria Optimization: Theory, Computation, and Applications. John Wiley \& Sons, Inc.

Swinehart, D. and Broek, D. (1995). Tenacity and fracture toughness of paper and board. Journal of pulp and paper science, 21(11):J389-J397.

Timoshenko, S. P. and Woinowsky-Krieger, S. (1959). Theory of plates and shells. New York : Tokyo : McGraw-Hill, 2nd edition. ISBN 0-07-085820-9.

Tryding, J. (1996). In-plane fracture of paper. Report tvsm-1008, Lund University, Lund Institute of Technology, Division of Structural Mechanics. Sweden.

Ulsoy, A. G. and Mote, C. D. (1982). Vibration of wide band saw blades. ASME Journal of Engineering for Industry, 104:71-78.

Vafai, A., Javidruzi, M., and Estekanchi, H. E. (2002). Vibration, buckling and dynamic stability of cracked cylindrical shells. Thin-Walled Structures, 40(1):29-44.

Valenzuela, M. A., Bentley, J. M., and Lorenz, R. D. (2009). Computer-aided controller setting procedure for paper machine drive systems. IEEE Transactions on Industry Applications, 45(2):638-650.

Wang, Y., Huang, L., and Liu, X. (2005). Eigenvalue and stability analysis for transverse vibrations of axially moving strings based on Hamiltonian dynamics. Acta Mechanica Sinica, 21:485-494. 
Wathén, R. (2003). Characterizing the influence of paper structure on web breaks. Licentiate thesis, Helsinki University of Technology, Department of Forest Products Technology. Espoo, Finland.

Westergaard, H. M. (1939). Bearing pressures and cracks. Journal of Applied Mechanics, 6:A49-A53.

White, D. J. (1990). A bibliography on the applications of mathematical programming multiple-objective methods. Journal of the Operational Research Society, 41(8):669-691.

Wickert, J. A. and Mote, C. D. (1988). Current research on the vibration and stability of axially-moving materials. Shock and Vibration Digest, 20:3-13.

Wickert, J. A. and Mote, C. D. (1990). Classical vibration analysis of axially moving continua. ASME Journal of Applied Mechanics, 57:738-744.

Yokoyama, T. and Nakai, K. (2007). Evaluation of in-plane orthotropic elastic constants of paper and paperboard. In 2007 SEM Annual Conference 83 Exposition on Experimental and Applied Mechanics. 


\section{List of Figure Captions}

Figure 1. A travelling web having an initial crack, and being supported by a system of rollers.

Figure 2. Two examples of cyclic tension. There may be few or many tension cycles per span.

Figure 3. Behaviour of $\Phi$ and $\Psi$ as functions of $\gamma$.

Figure 4. An admissible length of the crack and the critical tension, a schematic figure. Note the assumption $a^{\text {cr }}=a^{*} \ll 2 b$.

Figure 5. Dependence of the critical number of cycles (dimensionless) on the average tension (dimensionless).

Figure 6. Critical tension (a) and the corresponding critical velocity (b) with respect to the critical crack length for two different values of the strain energy rate.

Figure 7. Critical crack length $a^{\mathrm{cr}}$ (a) and critical tension $T_{\max }^{\mathrm{cr}}$ (b) with respect to the initial crack length $a_{0}$ and the tension variation $\Delta T$. The number of cycles $n^{\text {cr }}=n_{\mathrm{C}}=100$ is fixed.

Figure 8. Pareto fronts for the problems $\max \left\{\tilde{J}_{\mathrm{N}}, \tilde{J}_{\mathrm{V}}\right\}, \max \left\{\tilde{J}_{\mathrm{M}}, \tilde{J}_{\mathrm{V}}\right\}$, and $\max \left\{\tilde{J}_{\mathrm{M}}, \tilde{J}_{\mathrm{N}}\right\}$, respectively, in the case when the initial crack length $a_{0}=0.01 \mathrm{~m}$.

Figure 9. Dependence of the optimal tension $T_{0}$ on the parameter $a_{0}$ and the weight $C_{\mathrm{N}}$ 
or $C_{\mathrm{M}}$. 